\title{
Analysis and Design of Intze Water Tank by Using STAAD Pro
}

\author{
Chandana Imadabathun $i^{1}$, Padala Sri Vardhan Goud ${ }^{2}$, Nalla Ravi Kiran ${ }^{3}$, Bathula Naveen ${ }^{4}$ \\ ${ }^{1}$ Assistant Professor, Dept. of civil Engineering, Gokaraju Rangaraju Institute of Engineering and Technology, Telangana. \\ ${ }^{2}$ Student civil Engineering Department, GRIET, Hyderabad, Telangana, India. \\ ${ }^{3}$ Student civil Engineering Department, GRIET, Hyderabad, Telangana, India. \\ ${ }^{4}$ Student civil Engineering Department, GRIET, Hyderabad, Telangana, India.
}

\begin{abstract}
Water tank is a water storage structured built for long term use. These tanks were utilized for various uses like distribution of water, firefighting, agriculture, food industry, paper mills etc. It comes in handy when there is an intermittent supply of water or scarcity of water. Materials like concrete, pvc Galvanized Iron, fibre is used to manufacture tanks. Water is pumped through pipe by using pumps from a source. For distribution purpose water can be distributed either gravity or pump to reach individual with desired pressure and velocity. Volume is calculated based upon population and their usage and demand. Water demand varies hour to hour. For a continues supply water tanks are best suited. To meet water demand by public water tanks are to be constructed. Design and analysis are similar for any liquid present in water tank but is should be crack free to avoid leakage
\end{abstract}

\section{Introduction}

Overhead tanks and reservoirs are liquid storage containers. These containers are generally used for storing water for irrigation works, human consumption, fire, manufacturing units, rainwater harvesting, and for many purposes. The main purpose of design of tanks are economical, strength, service life, to provide safe portable drinking water after storing for a long time and it also resist special conditions like wind and earthquakes. Water tanks are generally constructed with reinforced concrete or steel and design is based on IS code. Design of tanks depends on the position of tank i.e, above or below, at the ground level. The overhead tanks are generally constructed at certain height from the ground level using columns and braces, for direct distribution of water by gravity. In any case, the underground tanks are rest underneath the ground level.

\section{Effect of Wind}

The wind pressure acting on a Design structure are processed by method suggested as per the IS code.

Design wind speed $\left(V_{Z}\right)$ at any elevation can be determined as follows:

$$
V_{z}=V_{b} \times k_{1} \times k_{2} \times k_{3}
$$

Where, $V_{z}=$ Design wind velocity for elevation ' $z$ ' in $\mathrm{m} / \mathrm{sec}$

$V_{b}=$ Basic wind speed based on locations.

$k_{1}=$ Risk coefficient or Probability factor

$k_{2}=$ elevation $\&$ construction, Terrain size factor and

$k_{3}=$ Topography factor

$k_{1}, k_{2}$ and $k_{3}$ are determined through IS 875 (Part-3) 2015. The design wind pressure for elevation above mean ground level will be acquired by the accompanying connection between wind pressing factor $\&$ wind speed

$$
P_{Z}=\left(0.6 V_{Z}^{2}\right)
$$

Where, $P_{Z}$ is Design Wind pressure $\left(\mathrm{N} / \mathrm{m}^{2}\right)$ at elevation Z,

$V_{z}$ is Design wind speed $(\mathrm{m} / \mathrm{s})$ elevation $\mathrm{z}$

\section{Intze tank}

A German hydraulic engineer is given the name tank as Intze. The water tower built in accordance with the Intze precept has a brick shaft on which the water tank sits. the bottom of the tank is fixed with a hoop anchor (Ring Anker) manufactured from iron or metal, so that 
handiest vertical, no longer horizontal, forces are transmitted to the tower. In this project Intze tank is designed with working stress method and elements of Intze tank is designed with limit state method.

Components of Intze tank:

Top dome: In general, we provide $100 \mathrm{~mm}$ to $150 \mathrm{~mm}$ thick dome at top with reinforcement along the latitudes and longitudes. Usually, the rise of the dome is $1 / 5$ th of the length.

Top ring beam: The top ring beam is subjected to meridional thrust. The beam is design for hoop tension which caused due to water load.

Cylindrical walls: The wall is mainly subjected to hoop tension which is caused by the water pressure. So, the wall is designed for hoop tension.

Bottom ring beam: This ring beam provided between the cylindrical wall and conical slab. The ring beam is provided to resist the horizontal component of the reaction of the conical wall on the cylindrical wall. The bottom ring beam is designed for the induced hoop tension.

Conical slab: The slab is subjected to both meridional thrust and hoop tension. The hoop tension is due to fluid pressure and meridional is due to vertical pressure. So, the slab should be designed safely.

Bottom dome: The bottom slab may be circular or domed. This slab is supported on the circular girder

Circular girder: The girder should be designed for the loads which are coming from the conical slab (inclined thrust) and from the bottom dome (outward thrust). It will be placed on the columns and should be designed for resulting bending moment and torsion.

Columns: The columns are designed for the total transmitted from the tank. Columns are also designed for wind and earthquake loads. In columns, bracings are provided at intervals to resist the wind and earthquake effects.

Foundations: In general, to support the all columns combined footing is adopted. To support a circular girder and circular slab are design.

\section{METHODOLOGY}

\section{Manual design}

Capacity of Intze water tank $=0.3 \mathrm{ML}$

$\mathrm{SBC}$ of soil $200 \mathrm{kN} / \mathrm{m}^{2}$

Wind pressure as IS $875-1200 \mathrm{~N} / \mathrm{m}^{2}$

Assuming $M_{30}$ concrete,

For which

$\sigma_{c b c}=10 \mathrm{~N} / \mathrm{mm}^{2}, \sigma_{c c}=8 \mathrm{~N} / \mathrm{mm}^{2}$
Tension in bending $=2.0 \mathrm{~N} / \mathrm{mm}^{2}$

Modular ratio $=\frac{280}{3 \sigma_{c b c}}=9.33$

For Rebar stress,

Indirect tension $($ Tensile stress $)=115 \mathrm{~N} / \mathrm{mm}^{2}$

Tensile stress (bending) for liquid face $=115 \mathrm{~N} / \mathrm{mm}^{2}$ for $\mathrm{t}$ less than $225 \mathrm{~mm}$

Volume $=0$. sos $\nu^{-}$ior (as per) ngure

$\mathrm{D}=8.0 \mathrm{~m}$

\section{Design of Top Dome:}

Assuming the rise $=1.5 \mathrm{~m}$

Therefore radius is given by

$1.5(2 * \mathrm{R}-1.50)=\left(\frac{8}{4}\right)^{2}$

$\mathrm{R}=6.08 \mathrm{~m}$

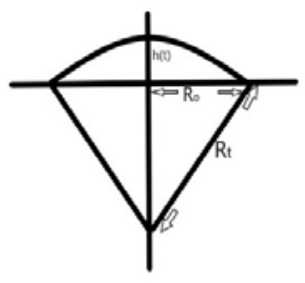

Fig 2. TOP DOME

$\operatorname{Sin} \emptyset=\frac{4}{6.08}=0.6579$

$\operatorname{Cos} \emptyset=0.7531$ and

$\emptyset=41.14^{\circ}<51.8^{\circ}$ (Therefore no tension).

Self-weight $=2.4 \mathrm{kN} / \mathrm{mm}^{2}$

Live load $=1.5 \mathrm{kN} / \mathrm{mm}^{2}$

Finishes $=0.10 \mathrm{kN} / \mathrm{mm}^{2}$

Total load $=5.2 \mathrm{kN} / \mathrm{mm}^{2}$

$\mathrm{f}=\frac{W R}{t}\left(\cos \emptyset-\frac{1}{1-\cos \emptyset}\right)$

$=5200 * \frac{6.08}{10}\left(0.75-\frac{1}{1.75}\right)$

$\mathrm{f}=0.0564 \mathrm{~N} / \mathrm{mm}^{2}$

hoop stress at crown, $\varnothing=0^{\circ}$

$\mathrm{f}=4950 * \frac{6.08}{0.1}\left(1-\frac{1}{2}\right)$

$\mathrm{f}=0.15 \mathrm{~N} / \mathrm{mm}^{2}$

$\mathrm{T}=\frac{W R}{1+\cos \varnothing}=4950 * \frac{6.08}{1+0.75}=17197.71 \mathrm{~N} / \mathrm{mm}^{2}$ 
Compressive stress $=\frac{17197.71}{100 * 1000}=0.172 \mathrm{~N} / \mathrm{mm}^{2}$

\section{Top Ring Beam :}

Horizontal component of $\mathrm{T}=\cos \emptyset$

$$
\begin{aligned}
& =17197.71 * 0.7531 \\
& =12951.59 \mathrm{~N} / \mathrm{m}
\end{aligned}
$$

Hoop stress in the ring beam $=12951.59 \times 8 / 2$

$$
=51806.38 \mathrm{~N}
$$

Area of steel required $=51806.38 / 115=450.49 \mathrm{~mm}^{2}$

Provide 4 bars of $12 \mathrm{~mm}$ diameter

$A_{\text {st }}$ provided $=452.39 \mathrm{~mm}^{2}$

Dimensions of the ring beam:

Let cross sectional area $=\mathrm{A} \mathrm{mm}^{2}$

Equivalent concrete area $=A_{c}+(9.33-1) A_{s t}$

$$
=A_{c}+8.33 * 452.39
$$

Concrete Cross sectional area,51806.38/2 $=43171.98$ $\mathrm{mm}^{2}$

Use a ring beam of size $230 \mathrm{~mm} \times 200 \mathrm{~mm}$

Area provided $=46000 \mathrm{~mm}^{2}>43565.63 \mathrm{~mm}^{2}$

Use $6 \mathrm{~mm}$ nominal stirrups at $100 \mathrm{~mm} \mathrm{c} / \mathrm{c}$

Shear stress along edge $=\mathrm{T} \sin \emptyset=17197.71 \times 0.6579=$ $11314.37 \mathrm{~N}$

Shear stress $=11314.37 / 100 * 1000=0.113 \mathrm{~N} / \mathrm{mm}^{2}$

\section{Design of cylindrical wall}

Pressure intensity $=5 \times 9810=49050 \mathrm{~N} / \mathrm{mm}^{2}$ (at the bottom of cylindrical wall)

Hoop tension $=49050 \times 8 / 2=196200 \mathrm{~N}$

$A_{s t}=\frac{196200}{230 \times 0.9}=947.83 \mathrm{~mm}^{2}$

Provide 10 nos of $12 \mathrm{~mm}$ dia bars

Area provided $=1130.97 \mathrm{~mm}^{2}$

Spacing of $12 \mathrm{~mm}$ dia bar $=1000 \times \pi \frac{x \frac{12^{2}}{1130.97}}{2}=200 \mathrm{~mm}$

Hence provide $12 \mathrm{~mm}$ at $200 \mathrm{~mm} \mathrm{c} / \mathrm{c}$ spacing at both directions

Assume thickness of wall $=230 \mathrm{~mm}$

Distribution steel $=\frac{0.24}{100}(230 \times 1000)=552 \mathrm{~mm}^{2}$

Provide $10 \mathrm{~mm}$ dia rebars of spacing $100 \mathrm{~mm}$ b/w the compressive stress check (at the bottom of the cylindrical wall)

Vertical component of $T_{1}=V_{1}=T_{1} \sin \emptyset=17197.71 \mathrm{x}$ $\sin 41.14$

\section{$11314.37 \mathrm{~N} / \mathrm{mm}^{2}$}

Weight of the wall $=0.23 \times 5 \times 25000=28750 \mathrm{~N} / \mathrm{m}$

Ring beam self-weight $=0.23 \times 0.2 \times 25000=$ $1150 \mathrm{~N} / \mathrm{m}$

Total load $V_{2}=41214.37 \frac{\mathrm{N}}{\mathrm{m}}$

Compressive stress $=\frac{41214.37}{230 \times 1000}=0.18 \mathrm{~N} / \mathrm{mm}^{2}$

Nominal vertical stress is equal to $0.24 \%$ gross area

$$
=\frac{0.24}{100} \times 230 \times 1000=552 \mathrm{~mm}^{2}
$$

Provide 12 bars of $8 \mathrm{~mm}$ diameter of spacing $100 \mathrm{~mm}$ $\mathrm{c} / \mathrm{c}$

\section{Bottom Ring Beam}

Let $T_{2}=$ Thrust $/$ meter exerted by the conical wall at the junction bottom at $\mathrm{B}$

$T_{2} \sin \propto=V_{2}$

$\tan \propto=1$

$\propto=45^{\circ}$

$T_{2}=\frac{V_{2}}{\sin \alpha}=\frac{41214.37}{\sin 45}=58285.92 \mathrm{~N} / \mathrm{mm}^{2}$

Resolving horizontally at B

$H_{2}=T_{2} \cos \alpha=58285.92 \times \cos 45^{\circ}$

$$
=41214.37 \mathrm{~N} / \mathrm{m}
$$

The lateral load $\left(\mathrm{H}_{2}\right)$ will create hoop tension in the ring - beam B

Hoop tension through $H_{2}=H_{2} \times \frac{d}{2}$

$$
\begin{aligned}
& =41214.37 \times \frac{8}{2} \\
& =164857.48 \mathrm{~N}
\end{aligned}
$$

Assume the depth of ring beam be $500 \mathrm{~mm}$ deep

Pressure due to water at ring beam $=9810 \times 5 \times \frac{500}{1000}$

$$
=24525 \mathrm{~N} / \mathrm{m}
$$

Hoop tension due to water $=24525 \times \frac{8}{2}=98100 \mathrm{~N}$

Total hoop tension $=98100+164875.48=262957.48 \mathrm{~N}$

Steel for hoop tension $=\frac{262957.48}{230 \times 0.9}=1270.32 \mathrm{~mm}^{2}$ 
Provide 6 bars of $18 \mathrm{~mm} \emptyset$

$A_{s t}=1526.81 \mathrm{~mm}^{2}$

Net concrete area $=A_{c}+(9.33-1) A_{s t}=A_{c}+8.33 *$ 1526.81

$$
=A_{c}+12718.33
$$

Limiting tensile stress for net concrete area $=2 \mathrm{~N} / \mathrm{mm}^{2}$

$$
\begin{gathered}
\frac{262957.48}{A_{c}+12718.33}=2 \\
A_{c}=118760.41
\end{gathered}
$$

Provide $250 \mathrm{~mm} \times 500 \mathrm{~mm}$ size

\section{Conical Slab}

$$
\frac{W_{w}+W_{s}}{2 \pi}+\frac{W_{w}}{2 \pi} \tan \alpha
$$

Where,

$W_{w}=$ weight of water resting on conical slab

$W_{s}=$ weight of the conical slab

$\alpha=$ slop of conical slab

$$
=\frac{6.5+5}{2} \times 8.625 \mathrm{~m}^{2}
$$

$\bar{X}=\frac{[6.5+(2 \times 3)]}{6.5+5} \times \frac{1.5}{3}=0.72 \mathrm{~m}$

Self -weight of water

$W_{w}=9810 \times 8.625 \times 2 \pi(2.5+0.72)=2.121 \mathrm{~m}$

Length of the conical slab $=2121 \mathrm{~mm}$

Thickness of the slab as $200 \mathrm{~mm}$

Self- weight $W_{s}=0.2 \times 2.121 \times 2500 \times 2 \pi \times \frac{6.5}{2}=$ $216557.83 \mathrm{~N}$

Hoop tension $=\frac{1711842.68+216557.83+1711842.68}{2 \pi}=$ $579362.697 \mathrm{~N}$

Hoop steel on the entire section $=\frac{579362.697}{230 \times 0.9}=$ $2798.85 \mathrm{~mm}^{2}$

Provide 14 bars of $16 \mathrm{~mm}$ diameter

$A_{s t}=2814.86 \mathrm{~mm}^{2}$

\section{Design for Flexure}

Load $/$ meter width of conical slab $=\frac{W_{w}+W_{S}}{2 \pi \times \text { mean radius }}=$ $\frac{1711842.68+216557.83}{2 \pi \times 3.25}=94435.22 \mathrm{~N}$

Maximum bending moment $=\frac{w l}{8}=\frac{94435.22 \times 1.5}{8}=$ $17706.604 \mathrm{Nm}$
Axial compression $V_{2}=T_{2} \sin \alpha$

$T_{2}=\frac{41214.37}{\sin 45^{\circ}}=5285.92 \mathrm{~N}$

Provide $16 \mathrm{~mm}$ rebar, at the clear cover $=25 \mathrm{~mm}$

Effective depth $=200-25-8=167 \mathrm{~mm}$

Distance between center of section (x) $=d-\frac{t}{2}=167$ $100=67 \mathrm{~mm}$

Resultant bending moment $=M+T_{2} x=17706.6040 \times$ $10^{3}+58285.92 \times 67$

$$
=21611760.64 \mathrm{~N}-\mathrm{m}
$$

$A_{s t}=\frac{21611760.64}{167 \times 230 \times 0.9}=625.177 \mathrm{~mm}^{2}$

Provide 4 no's bars, $16 \mathrm{~mm}$ diameter

\section{Design of bottom dome}

$\mathrm{R}$ is the radius of dome, rise $=1.2 \mathrm{~m}$

Then, $(2.5)^{2}=1.2(2 R-1.2)$

$$
\mathrm{R}=3.20 \mathrm{~m}
$$

Let $2 \theta$ be the angle subtended by the dome

$\operatorname{Sin} \theta=\frac{2.5}{3.2}=0.78$,

$\theta=51.37^{\circ}<51.8^{\circ}$

$\operatorname{Cos} \theta=0.624$

Thickness of dome $=0.2 \mathrm{~m}$

Loads:

Dead load $=25000 \times 0.2=5000 \mathrm{~N} / \mathrm{mm}^{2}$

Weight of water resting on the dome $=r_{w}\left(\pi r^{2}-\right.$ $\frac{\pi h_{c}}{3}\left(3 R-h_{c}\right)$

$$
\begin{gathered}
=9810\left(\pi \times 2.5^{2}-\frac{\pi \times 1.2}{3}(3 \times 3.2-1.2)\right) \\
=1127757.6 \mathrm{~N}
\end{gathered}
$$

Area of dome surface $=2 \pi R h=2 \times \pi \times 3.2^{2} \times 1.2$

$$
=24.13 \mathrm{~m}^{2}
$$

Load intensity due to weight of water $=\frac{1127757.6}{24.13}=$ $46736.74 \mathrm{~N} / \mathrm{mm}^{2}$

Meridional thrust $=\frac{W R}{1+\cos \theta}=46736.74 \times \frac{3.2}{1+0.624}$

$$
=92092.098 \mathrm{~N} / \mathrm{m}
$$


Meridional compressive stress $=\frac{92092.098}{200 \times 1000}=0.46 \frac{\mathrm{N}}{\mathrm{mm}^{2}}$

Hoop stress at the crown $\theta=0^{\circ}$

Hoop stress $=\frac{W R}{t}\left(\cos \theta-\frac{1}{1+\cos \theta}\right)=46736.74 \times$ $\frac{3.2}{0.2}\left(0.624-\frac{1}{1+0.624}\right)$

$=0.006 \frac{\mathrm{N}}{\mathrm{mm}^{2}}$

Max. hoop stress $=\frac{W R}{t}\left(\cos \theta-\frac{1}{1+\cos \theta}\right)=$ $46736.74 \times \frac{3.2}{0.2}\left(1-\frac{1}{2}\right)$

$$
=0.374 \mathrm{~N} / \mathrm{mm}^{2}
$$

Provide nominal $0.3 \%$ of steel

Provide $8 \mathrm{~mm}$ diameter bars at $100 \mathrm{~mm} \mathrm{c} / \mathrm{c}$ spacing

\section{Design circular girder:}

Total water weight $w_{1}=$ conical slab water weight + dome water weight

\section{$=1711842.68+1127757.6=2839600.28 \mathrm{~N}$}

Weight of the dome + cylindrical wall + ring beam at A $W_{2}=41214.37 \times 2 \pi \times 4$

$$
=1035830.096 \mathrm{~N}
$$

Weight of ring beam at B $W_{3}=0.25 \times 0.5 \times 25000 \times$ $2 \pi \times 4=78539.82 \mathrm{~N}$

Weight of conical wall $W_{4}=216557.83 \mathrm{~N}$

Weight of lower dome $W_{5}=5000 \times 24.13$

$$
=120650 \mathrm{~N}
$$

Weight of circular girder $=0.4 \times 0.6 \times 25000 \times 2 \pi \times$ 2.5

$($ Assume size of girder $100 \times 600)=94247.78 \mathrm{~N}$

Total load $(\mathrm{W})=4385425.806 \mathrm{~N}$

Let us provide 8 columns

Maximum bending moment at supports $=0.0083 \mathrm{Wr}$

$$
\begin{aligned}
& =0.0083 \times 43854525.806 \times 2.5 \\
& =90997.585 \mathrm{Nm}
\end{aligned}
$$

Maximum bending moment at center $=0.00416 \mathrm{Wr}$

$=0.0006 \times 4385425.806 \times 2.5$

$=6578.139 \mathrm{Nm}$

Shear force at supports $=\frac{W}{2 \times \text { numbers of columns }}=$ $\frac{4385425.806}{2 \times 8}$

$$
=274089.11 \mathrm{~N}
$$

Load at each support $=\frac{W}{8}=\frac{4385425.806}{8}$

$$
=548178.22 \mathrm{~N}
$$

\section{Design of support section:}

Moment of resistance $=$ bending moment at support

$$
\begin{gathered}
0.913 b d^{2}=90997.585 \times 1000 \\
d=499.17 \mathrm{~mm}
\end{gathered}
$$

Clear cover $=40 \mathrm{~mm}$, adopt $\mathrm{D}=550 \mathrm{~mm}, \mathrm{~d}=510 \mathrm{~mm}$.

Equivalent shear force $=V+\frac{1.6 T}{b}$

$$
\begin{aligned}
& =274089.11+\frac{1.6 \times 6578.139 \times 1000}{400} \\
& V_{c}=300401.66 \mathrm{~N}
\end{aligned}
$$

Equivalent nominal shear stress $\tau_{v c}=\frac{V_{c}}{b d}=\frac{300401.66}{400 \times 510}$

$$
=1.47 \frac{\mathrm{N}}{\mathrm{mm}^{2}}
$$

Maximum shear stress $\tau$ max $>\tau_{v}$

$$
\begin{aligned}
& \tau \max =2.2 \mathrm{~N} / \mathrm{mm}^{2} \\
& \tau_{c}<\tau_{v}
\end{aligned}
$$

Longitudinal reinforcement:

$$
\begin{aligned}
& M_{e}=M+M_{t} \\
& M_{t}=\frac{T\left(1+\frac{D}{b}\right)}{1.7}=6578.139 \times \frac{1000\left(1+\frac{550}{400}\right)}{1.7} \\
&=9190047.132 \mathrm{Nmm} .
\end{aligned}
$$

$\mathrm{M}=$ moment at cross section

$$
\begin{aligned}
M_{e} & =90997.585 \times 1000+9190047.132 \\
& =100187632.1 \mathrm{Nm}
\end{aligned}
$$

$$
\begin{array}{r}
A_{s t}=\frac{M_{e}}{230 \times 0.9 \times d}=\frac{100187632.1}{230 \times 0.9 \times 510} \\
=949.02 \mathrm{~mm}^{2}
\end{array}
$$

Transverse reinforcement:

$$
\begin{aligned}
& 550-80=470 \mathrm{~mm} \\
& A_{s v}=s_{v}\left(\frac{6578139}{320 \times 470 \times 230}+\frac{274089.11}{2.5 \times 470 \times 230}\right)
\end{aligned}
$$$$
A_{s v}=\frac{T s_{v}}{b_{1} d_{1} \sigma_{s v}}+\frac{V s_{v}}{2.5 d_{1} \sigma_{s v}}
$$$$
b_{1}=400-80=320 \mathrm{~mm}, d_{1}=
$$

Providing 4-legged $10 \mathrm{~mm}$ stirrups, $A_{s v}=315 \mathrm{~mm}^{2}$

Therefore $s_{v}=261.55 \mathrm{~mm}$, take $s_{v}$ as $250 \mathrm{~mm}$ 


$$
\begin{gathered}
A_{s v}=\left(\tau_{v c}-\tau_{c}\right) s_{v} \cdot \frac{b}{\sigma_{s v}}=\frac{1.37-0.2}{400} \times 400 \times s_{v} \\
s_{v}=154.81 \mathrm{~mm}
\end{gathered}
$$

Provide $150 \mathrm{~mm}$ spacing c/c.

Steel due to sagging moment $=45608.428 \times$ $\frac{1000}{230 \times 0.9 \times 510}=432.02 \mathrm{~mm}^{2}$

Provide 4 bars of $12 \mathrm{~mm}$ diameter

$$
A_{s t}=452.39 \mathrm{~mm}^{2}
$$

Hoop stress:

$T_{c}=$ thrust on girder acted by conical slab

$T_{c} \sin \alpha \times 2 \pi r$

$=W_{w}+W_{s}$

+ weight of cylindrical wall and upper dome

$$
\begin{aligned}
T_{c} \sin \alpha \times 2 \pi r= & 17111842.68+216557.83 \\
& +1035830.096
\end{aligned}
$$

$$
T_{c}=
$$

$$
266874.52 \mathrm{~N}
$$

Horizontal component of $T_{c}=H_{1}=266874.52 \cos 45$

\section{$=188708.78 \mathrm{~N}$}

Horizontal component due to dome $H_{2}=92092.098 \times$ $\cos 51.37^{\circ}$

$$
\begin{array}{r}
=57492.06 \mathrm{~N} \\
H_{1}-H_{2}=131216.72 \mathrm{~N}
\end{array}
$$

Hoop stress $=131216.72 \times 2.5=328041.81 \mathrm{~N}$

Hoop compressive stress $=\frac{328041.81}{400 \times 550}=1.5 \mathrm{~N} / \mathrm{mm}^{2}$

\section{Columns:}

Actual load on each column at top $=\frac{4385425.806}{8}=$ $548178.22 \mathrm{~N}$

Let $\alpha=$ Angle made by column with vertical axis

$$
\begin{gathered}
\tan \alpha=\frac{1}{10}, \alpha=5^{\circ} 42^{\circ}, \sin \alpha=0.0995, \cos \alpha=\frac{10}{\sqrt{101}} \\
=0.995
\end{gathered}
$$

Actual length of column $=\sqrt{10^{2}+1^{2}}=10.05 \mathrm{~m}$

Providing 300mm x 300mm column size

Self - weight $=10 * 0.3 * 0.3 * 25000=22500 \mathrm{~N}$

Total load $=548178.22+22500=570678.22 \mathrm{~N}$

Axial load $=\frac{540678.22}{0.9950}=573545.95 \mathrm{~N}$

Tank full condition $=573545.95 \mathrm{~N}$
Weight of water tank $=\frac{2839600.28}{8}=$ $354950.035 \mathrm{~N}$ on each column

In case of empty tank

Load on column $=573545.95-354950.035$

$=218595.915 \mathrm{~N}$

Corresponding axial load $=\frac{218595.915}{0.995}=219694.387 \mathrm{~N}$

Let $A_{s c}=$ steel requirement without wind effect

Then $\quad c A_{c}+t A_{s c}=573545.95 \mathrm{~N}$

$5 \times A_{c}+190 \times A_{s c}=573545.95$

$A_{s c}=667.82 \mathrm{~mm}^{2}$

Min requirement of steel $=0.8 \%$ Gross area

$$
\frac{0.8}{100}(300 \times 300)=720 \mathrm{~mm}^{2}
$$

Provide 6 bars of $20 \mathrm{~mm}$ diameter $A_{s c}=1884.95 \mathrm{~mm}^{2}$

\section{Analysis for wind pressure:}

Take reduction factor as 0.7

Wind thrust on top dome \& cylindrical walls = $\left(5+\frac{1.5}{2}\right) \times 8.46 \times 1200 \times 0.7$

$=40861.8 \mathrm{~N}$ acting at $14.37 \mathrm{~m}$ from the base

Wind thrust on circular wall $=\frac{8.46+5.40}{2} \times 1.5 \times 1200 \times$ 0.7

$=8731.8 \mathrm{~N}$ acting at 10.75 from the base

Wind thrust on the circular girder $=0.6 \times 5.4 \times$ $1200 \times 0.7$

$$
=2721.6 \mathrm{~N} \text { acting at } 10 \mathrm{~m} \text { from the base }
$$

Wind thrust on columns \& bracings $=(5 \times 0 . \times 10 \times$ $1200 \times 0.7)+\left(3 \times \frac{6+8}{2} \times 0.3 \times 1200\right)$

$=20160 \mathrm{~N}$ acting at $5 \mathrm{~m}$ from the base

Total moments due to wind pressure at the base $=$ $(40861.8 \times 14.37)+(8731.8 \times 10.75)+(2721.6 \times$ $10)+(20160 \times 5)=809066.92 \mathrm{Nm}$

Vertical load on column by wind load $=\frac{M x}{\epsilon x^{2}}$

$$
\in x^{2}=2 \times 4^{2}+4\left(\frac{4}{\sqrt{2}}\right)^{2}=64 \mathrm{~m}^{2}
$$

Max. wind thrust in most leeward side \& the most windward side

$$
=809066.92 \times \frac{4}{64} \times \frac{4}{\sqrt{2}}
$$




$$
=35756.04 \mathrm{~N}
$$

Consider windward column 1

Dead load + wind load $=570678.22+50566.682$

$$
=621244.902 \mathrm{~N}
$$

Corresponding axial load $=\frac{621244.902}{0.9950}=624366.74 \mathrm{~N}$

Horizontal component of the axial force due to wind action $=2 \times 50566.682 \times 0.0995+4 \times 35756.04 \times$ $0.0995 \times \frac{1}{\sqrt{2}}=20125.54 \mathrm{~N}$

Actual horizontal force at base $=40861.8+8731.8+$ $2721.6+20160-20125.54$

$$
=52349.66 \mathrm{~N}
$$

Horizontal shear column $=\frac{52349.66}{8}=6543.71 \mathrm{~N}$

Maximum bending moment for the column = $6543.71 \times \frac{2.5}{2}=8179.63 \mathrm{Nm}$

\section{Column section:}

Axial load $=624366.74 \mathrm{~N}$

B.M. $=8179.63 \mathrm{Nm}$

Provide 300 x $300 \mathrm{~mm}$ size

Use 6 rebars, $20 \mathrm{~mm} \phi$ at effective cover of $50 \mathrm{~mm}$

$A_{s t}=1884.95 \mathrm{~mm}^{2}$

Net concrete area $=A_{c}+(m-1) A_{s t}$

$=(300 \times 300)+(8.33 \times 1885)=105702.05 \mathrm{~N}$

For net concrete section the polar moment of inertia

( $m A_{s t} \times$ effective depth from centre)

$$
=\frac{a^{4}}{12}+
$$

$$
\begin{aligned}
& =\frac{300^{4}}{12}+\left(8.33 \times 1885 \times(150-50)^{2}\right) \\
& =832.02 \times 10^{6} \mathrm{~mm}^{4}
\end{aligned}
$$

Equivalent moment of inertia about full section = $832 \times \frac{10^{6}}{2}=416.01 \times 10^{6} \mathrm{~mm}^{4}$

$$
\begin{aligned}
& \begin{array}{l}
\text { Direct } \\
\text { direct }
\end{array} \frac{\text { stress }}{\text { equivalent concrete area }}=\frac{\text { concrete }}{105702.05}=5.91 \mathrm{~N} / \\
& \mathrm{mm}^{2}
\end{aligned}
$$

Bending stress in concrete $=\frac{8179630}{416.01 \times 10^{6}} \times 150=$ $2.95 \mathrm{~N} / \mathrm{mm}^{2}$

Maximum stress $=5.91+2.95=8.86 \mathrm{~N} / \mathrm{mm}^{2}$

Design of braces:

Moment in braces $=2 \times$ moment for the column $\times$ $\sec 45^{\circ}$

$$
=2 \times 8179.63 \times \sqrt{2}=23135.49 \mathrm{Nm}
$$

Provide 300 x $300 \mathrm{~mm}$ beam

Area of steel $==\frac{23135490}{230 \times 0.9 \times 220}=508.02 \mathrm{~mm}^{2}$

Use 4 rebars of $18 \mathrm{~mm}$ diameter

S.F. for brace $=\frac{\text { bending moment for brace }}{\frac{1}{2} \text { span of brace }}$

Span of brace $=2 \times \frac{7.5}{2} \times \sin 22^{\circ} 30^{\circ}=2.678 m$

Shear force for brace $=\frac{23135.49}{0.5 \times 2.678}=17278.18 \mathrm{~N}$

Nominal shear stress $\tau_{v}=\frac{V}{b d}=\frac{17278.18}{300 \times 260}=0.22 \frac{\mathrm{N}}{\mathrm{mm}^{2}}$

Provide nominal stirrups, 2 legged - $10 \mathrm{~mm}$ diameter stirrups $200 \mathrm{~mm}$ spacing $\mathrm{c} / \mathrm{c}$

\section{FOUNDATION Design}

Axial load on column $=573545.95 \times 8=$ $4588367.6 \mathrm{~N}$

Self- weight of footing $(10 \%$ of column load $)=$ $458836.76 \mathrm{~N}$

Total load $=5047204.36 \mathrm{~N}$

$\mathrm{SBC}=200 \mathrm{kN} / \mathrm{mm}^{2}$.

Area $=\frac{\text { load }}{\text { safe bearing capacity }}=\frac{5047204.36}{200 \times 10^{3}}=25.24 \mathrm{~m}^{2}$

Provide outer diameter of $9.0 \mathrm{~m} \&$ inner diameter of 6.0 $\mathrm{m}$

$$
=\frac{\pi}{4} \times\left(9.0^{2}-6.0^{2}\right)=35.34 \mathrm{~m}^{2}
$$

Net intensity $=\frac{5047204.36}{35.34}=142.8286 \mathrm{kN} / \mathrm{mm}^{2}$ $\mathrm{mm}^{2}$, hence ok

$$
142.828686 \mathrm{kN} / \mathrm{mm}^{2}<20086 \mathrm{kN} /
$$

\section{Design at Circular Girder}

Maximum negative B.M. occurs at supports $83985.48 N-\mathrm{m}$

$$
=0.00416 \times 5047204.36 \times 4=
$$

Maximum positive bending $=0.0083 \times$ $5047204.36 \times 4=83985.480 \mathrm{~N}-\mathrm{m}$

Maximum torsion $=0.0006 \times W \times r=12113.29 \mathrm{~N}-$ $\mathrm{m}$

Maximum shear force at support $=\frac{5047204.36}{2 \times 8}=$ $315450.27 \mathrm{~N}$

\section{Design at Support Section:}

Moment of resistant $=$ maximum bending moment at support assume $b=500 \mathrm{~mm}$ 


$$
\begin{aligned}
& 0.913 b d^{2}=167567.18 \times 1000 \\
& d=605.86 \mathrm{~mm} \text {, adopt } 610 \mathrm{~mm}
\end{aligned}
$$

Equivalent shea stress,

$$
\begin{gathered}
V_{v}=V+1.6 \times \frac{T}{b}=315450.27+\frac{1.6 \times 12113.29 \times 1000}{500} \\
V_{v}=354212.8 \mathrm{~N} \\
\tau_{v}=\frac{V_{v}}{b d}=\frac{354212.8}{500 \times 610}=1.16 \mathrm{~N} / \mathrm{mm}^{2} \quad \text { but } \\
\tau_{c}=2.2 \mathrm{~N} / \mathrm{mm}^{2} \\
\text { Hence, } \tau_{c}<\tau_{v} .
\end{gathered}
$$

Longitudinal reinforcement:

$$
\begin{gathered}
M_{e}=M+M_{t} \\
M_{t}=\frac{T\left(1+\frac{D}{b}\right)}{1.7}=\frac{12113.29 \times 10^{3}\left(1+\frac{660}{500}\right)}{1.7}=16531078.12 \mathrm{~N}- \\
M_{e}=(167567.18 \times 1000+16531078.12) \\
M_{e}=184098.26 \times 10^{3} \mathrm{~N} \\
A_{s t}=\frac{184098.26 \times 10^{3}}{230 \times 0.9 \times 610}=1457.97 \mathrm{~mm}^{2}
\end{gathered}
$$

Provide 9 bars of $16 \mathrm{~mm}$ diameter bars

Hence $A_{\text {st required }}=1810 \mathrm{~mm}^{2}$.

Transverse reinforcement

$$
\begin{aligned}
& A_{s v}=\frac{T \cdot S_{v}}{b_{1} d_{1} \sigma_{s v}}+\frac{V \cdot S_{v}}{2.5 d_{1} \sigma_{s v}} \\
& A_{s v}=4 \times \frac{\pi}{4} \times 10^{2}=314.16 \mathrm{~mm}^{2}, b_{1}=500-80=
\end{aligned}
$$
$420 \mathrm{~mm}$,

$$
\begin{gathered}
d_{1}=660-120=540 \mathrm{~mm} \\
314.16=\left(\frac{12113.29 \times 10^{3}}{420 \times 540 \times 230}+\frac{315450.27}{2.5 \times 540 \times 230}\right) S_{v} \\
S_{v}=251.7 \mathrm{~mm}
\end{gathered}
$$

Let us provide $200 \mathrm{~mm}$ clear cover spacing

Steel for hogging moment $A_{s t}=665.13 \mathrm{~mm}^{2}$

Provide 4 bars of $16 \mathrm{~mm}$ diameter

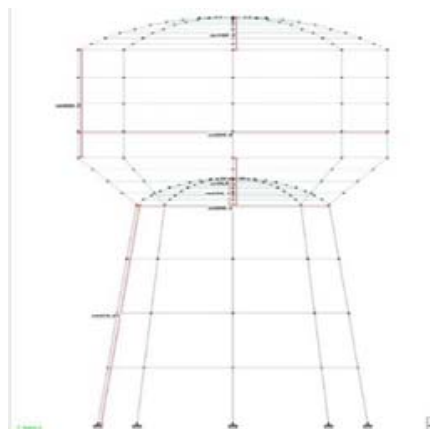

Fig 3. INTZE WATER TANK MODEL

\section{STAAD Pro design}

\section{Procedure}

1. Open STAAD.pro.

Click on new project $>$ add file name $>$ Select 'space'.

Length (in m), Force (in KN).

Select add beam option and click on finish.

2. Geometry $>$ Run structure wizard $>$ select surface/plate model $>$ cylindrical surface. Close it to transfer to modelling

Length :12

Division along length: 1

Start radius: 3.5

Division along periphery: 8 (column)

End radius: 2.5

3. Using Add beam selecting top node and bottom node. Repeat along periphery for required number of columns.

4. Copy all vertical members using $\mathrm{ctrl}+\mathrm{C}$ and paste aside using ctrl $+\mathrm{V}$.

5. Add intermediate nodes along length to add required number of beams in horizontal direction. Connect all node in a plane to form a circular beam.

6. Repeat the same process at top to get circular girder.

7. Geometry $>$ Run structure wizard $>$ select surface/plate model $>$ Spherical cube

Select spherical cap (Bottom dome). Close it to transfer to modelling

Diameter of sphere:

Base Diameter:

8. Shift the obtained Spherical cap to top beam Measure distance using 'display node to node distance' tool

Select all plates $>$ Right click mouse $>$ Move $>$ add (-) sign to above distance to rest on top beam.

1. Geometry $>$ Run structure wizard $>$ select surface/plate model $>$ cylindrical surface

Length: 2.12

Division along length: 1

Start radius: 35

Division along periphery: 8(column)

End radius: 2.5

2. Shift the obtained Conical dome to top beam Measure distance using 'display node to node distance' tool

Select all plates $>$ Right click mouse $>$ Move $>$ add (-) sign to above distance to rest on top beam.

3. Geometry $>$ Run structure wizard $>$ select surface/plate model $>\quad$ cylindrical surface

Length: 2.12

Division along length: 1

Start radius: 35

Division along periphery: 8(column)

End radius: 2.5

4. Shift the obtained cylindrical surface to top beam Measure distance using 'display node to node distance' tool

Select all plates $>$ Right click mouse $>$ Move $>$ add (-) sign to above distance to rest on top beam. 
5. Geometry $>$ Run structure wizard $>$ select surface/plate model $>$ Spherical cube

Select spherical cap (Top dome). Close it to transfer to modelling

Diameter of sphere:

Base Diameter:

6. Shift the obtained Conical dome to top beam Measure distance using 'display node to node distance' tool

Select all plates $>$ Right click mouse $>$ Move $>$ add (-) sign to above distance to rest on top beam.

7. Finally Check dimensions of tank using 'display node to node distance' tool to verify. Any corrections to be made are rectified.

\section{GENERAL PROPERTIES}

8. Click 'property' at left of screen> Define required dimensions for respective elements. Assign the property for various elements using any of the options present according to your convenient.

Click 'Support' $>$ Create $>$ Select 'fixed' $>$ click Add $>$ assign at bottom part of beam.

9. Click 'Load and Definition'

To apply wind load first we have to define it in first section. Enter your values. Keep exposure as -1 . Click 'Load case details' to add DL, LL \& WL.

Add self-weight as DL

Add Water load as LL

Add Wind Load

Select material as concrete and assign for entire tank

\section{Analysis}

10. Click 'Analysis and print' $>$ Run analysis $>$ Check for Zero errors $>$ Post processing

Apply given loads to see deflected shape of structure, beam moments and forces.

\section{Design}

11. Click on 'Design' $>$ Select parameters to include in our design.

Define parameters with respective values

Select the required command to instruct software to design according to IS code.

Detailing of reinforcement and quantity of concrete is present in output file.

\section{Design in STAAD Pro}

\section{Step 1: Geometry of Structure}
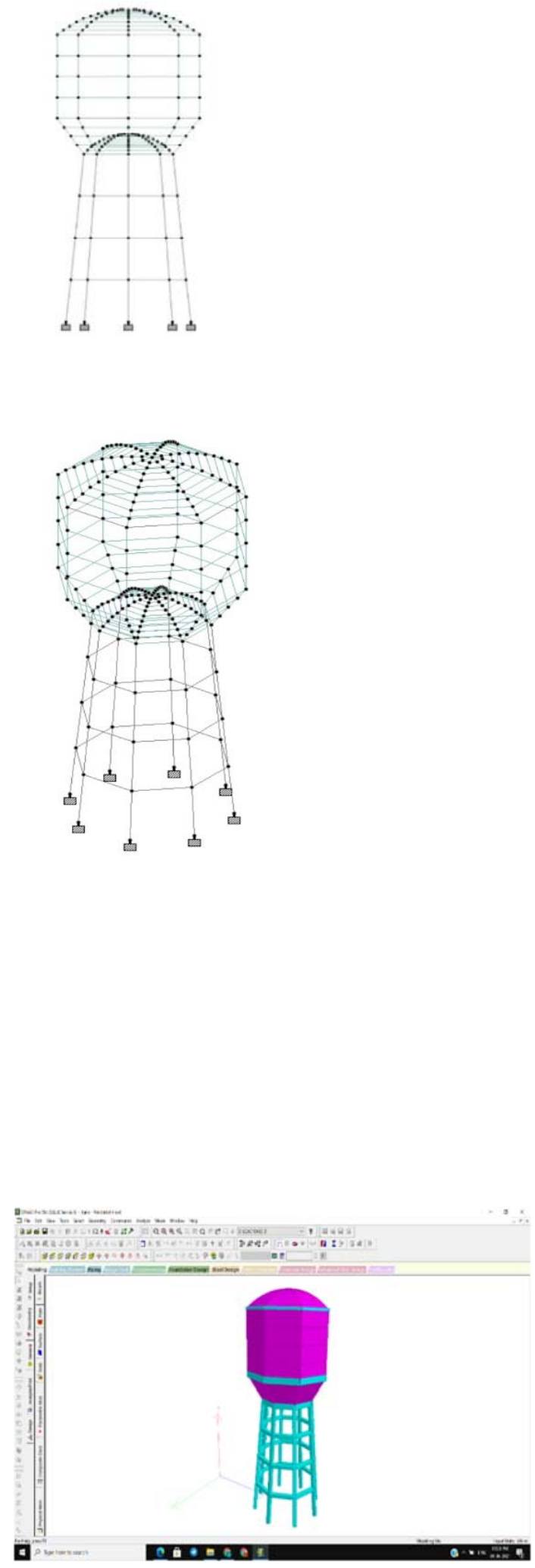
Step 2: Material and Property

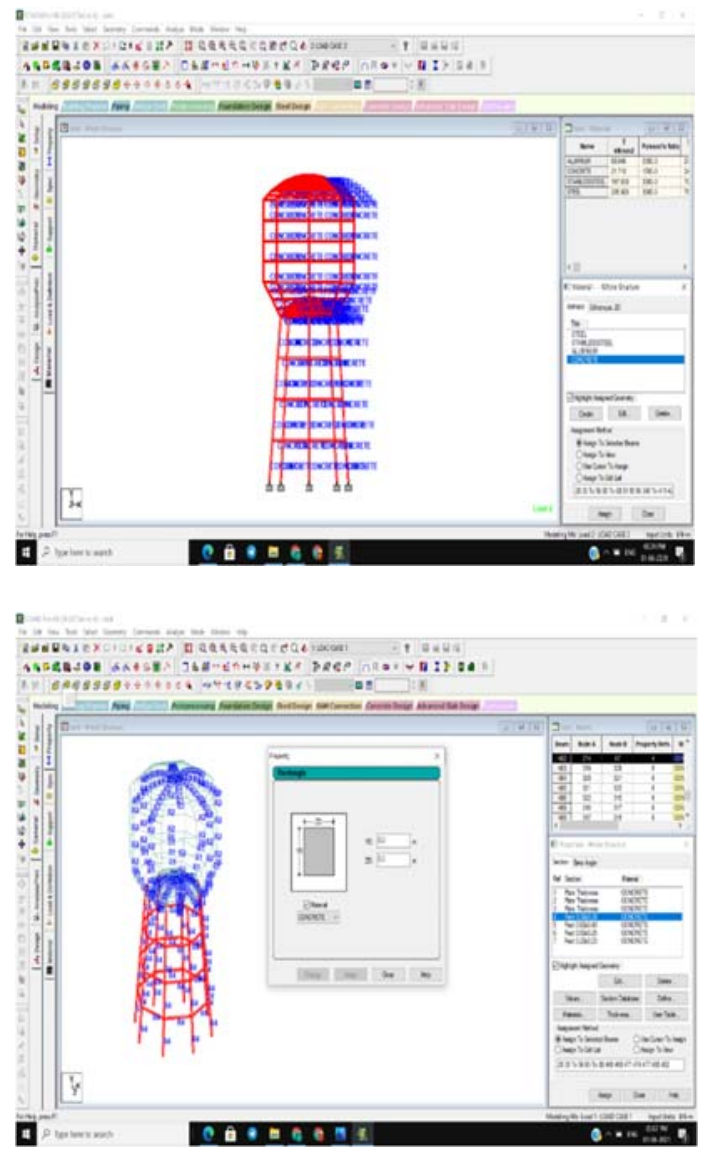

Step 3: Loads and Definitions

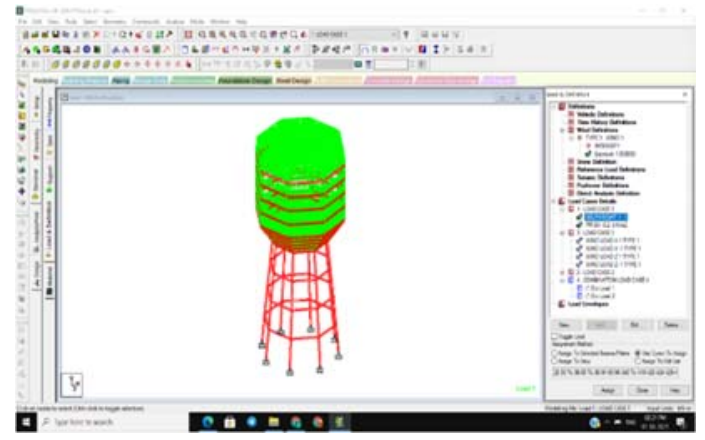

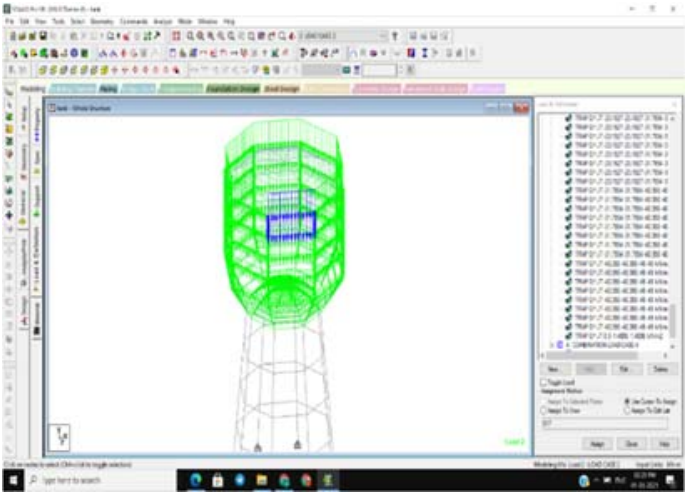

Step 4: Assign Support
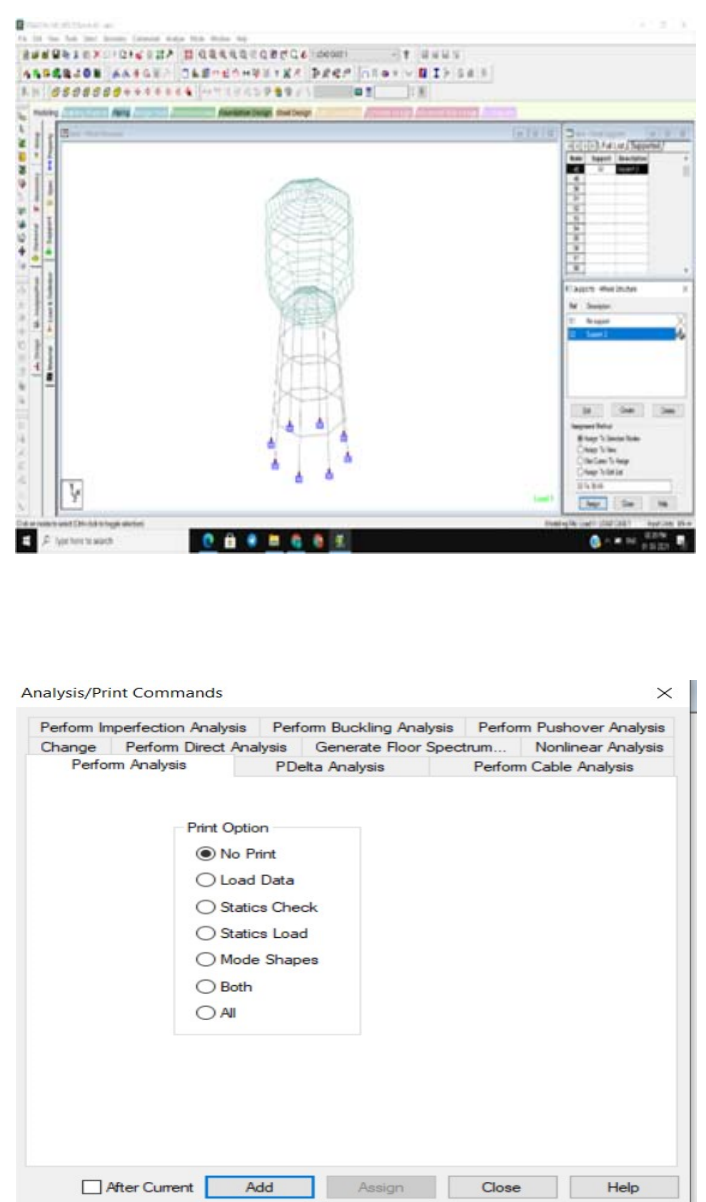

Step 5: Run Analysis 


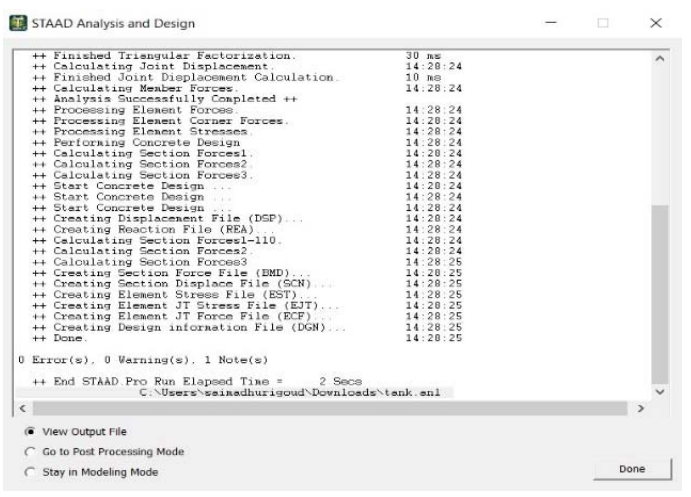

Step 6: Go to Post processing
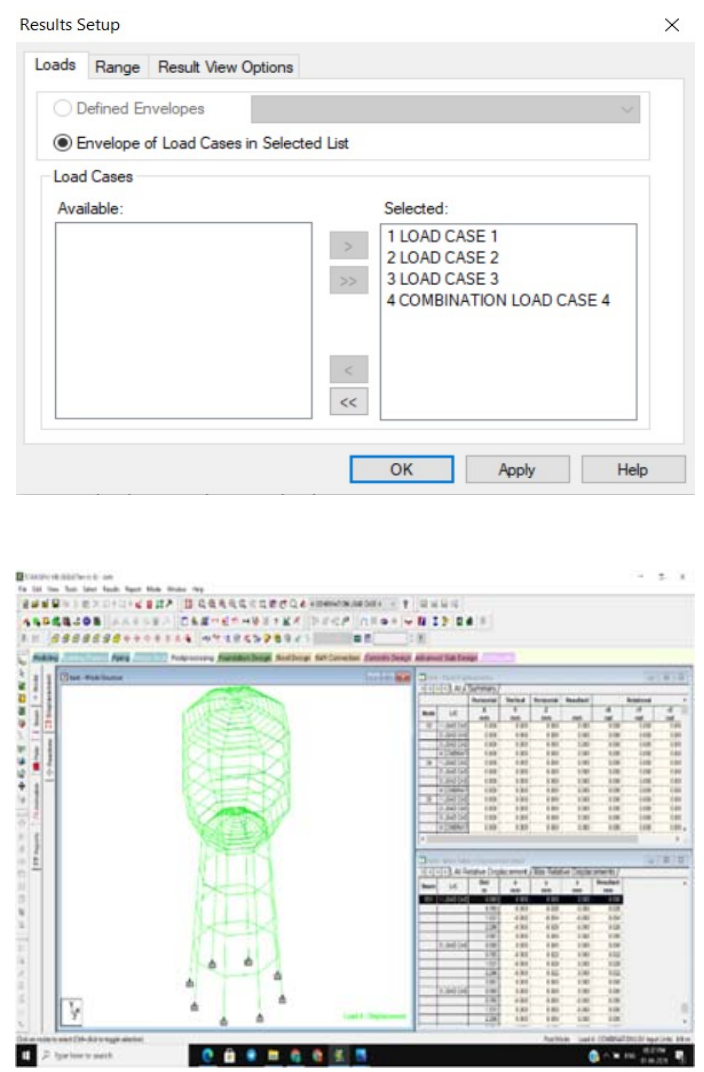

Step 7: Analysis Drawings

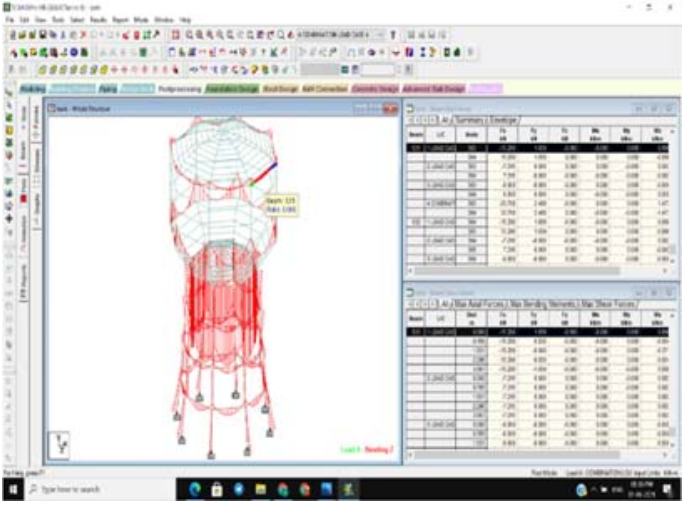

Bending moments of beam

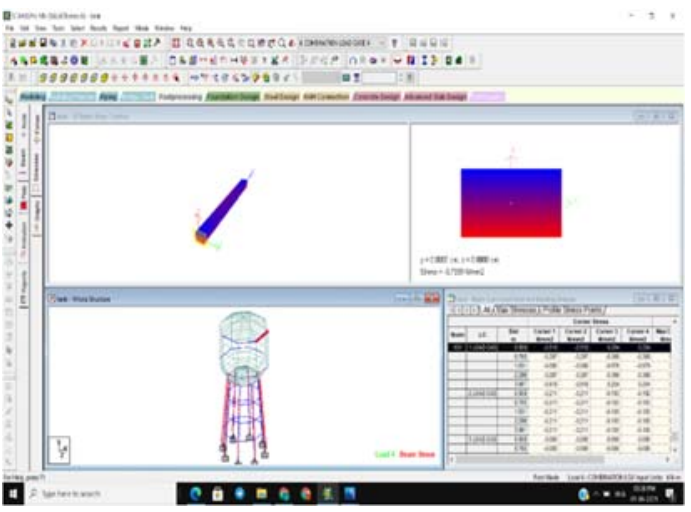

Stresses in Beam

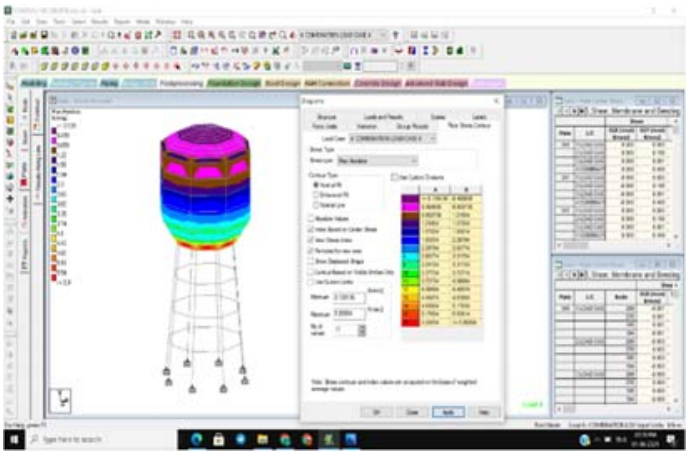

Max absolute and max Principal stress

\section{Step 8: Design}

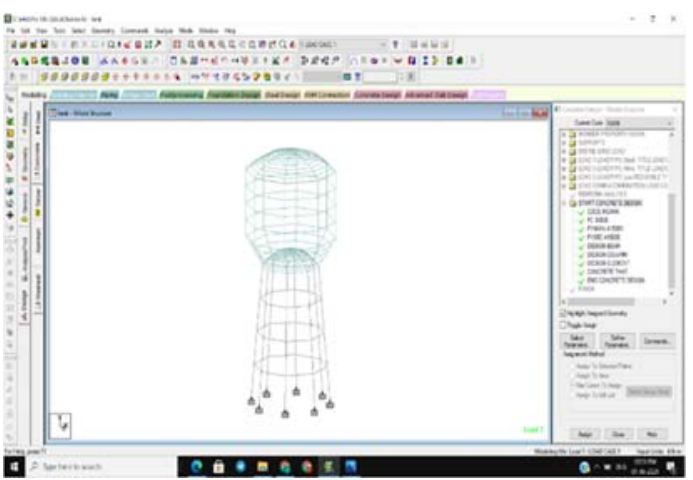




\section{Reinforcement Details}
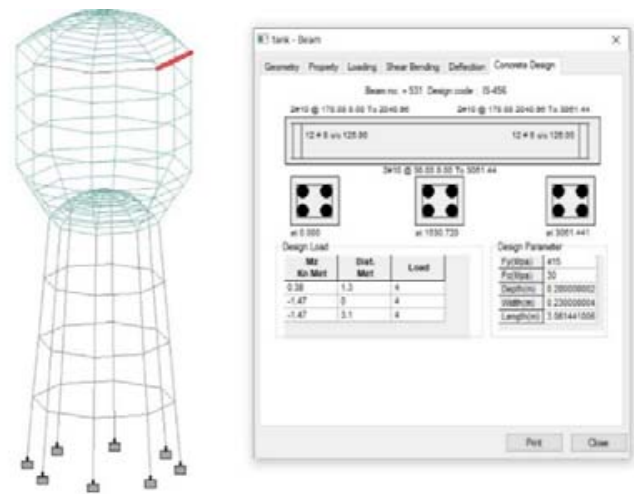

\section{Ring Beam}
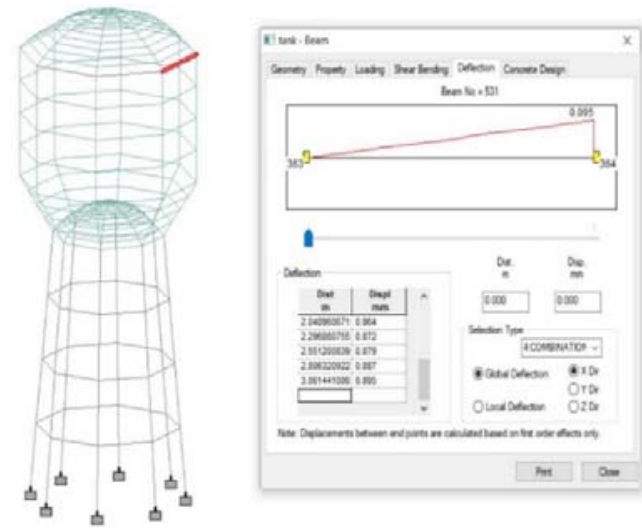

\section{Columns:}
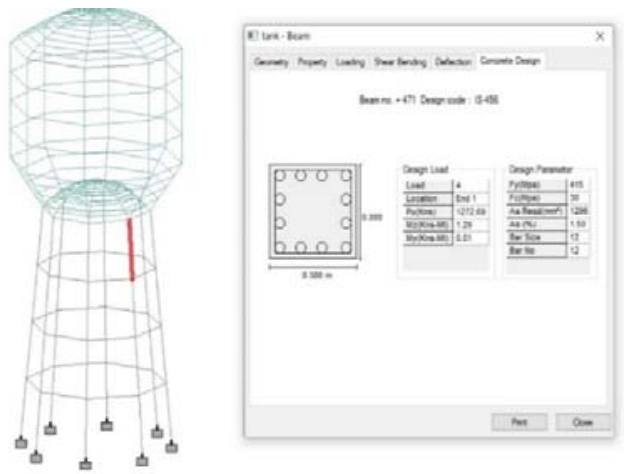
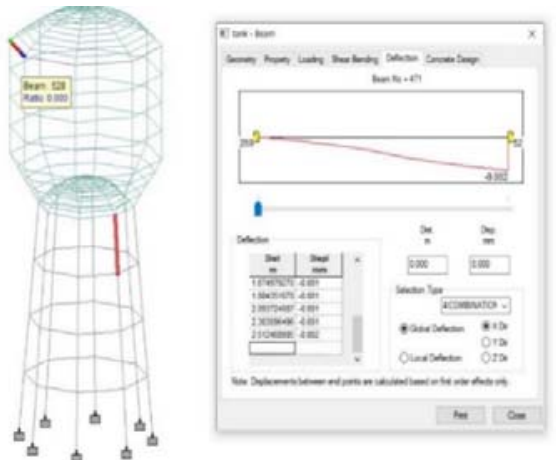

\section{RESULTS}

Manual design of Intze tank as per IS: $\mathbf{3 3 7 0}$

\section{Top dome:}

Thickness of the dome $=100 \mathrm{~mm}$ Force $=5.2 \mathrm{kN} / \mathrm{mm}^{2}$

Hoop stress $=0.15 \mathrm{~N} / \mathrm{mm}^{2}$

Meridional stress $=0.17 \mathrm{~N} / \mathrm{mm}^{2}$

Area of steel $=300 \mathrm{~mm}^{2}$

\section{Top ring beam:}

Size $=230 \times 200 \mathrm{~mm}$

Meridional thrust $=12.95 \mathrm{kN}$

Hoop tension $=51.81 \mathrm{kN}$

Shear stress $=0.113 \mathrm{~N} / \mathrm{mm}^{2}$

Area of steel $=452.39 \mathrm{~mm}^{2}$

\section{Cylindrical wall:}

Height $=4 \mathrm{~m}$

Thickness of wall $=230 \mathrm{~mm}$

Hoop tension $=196.2 \mathrm{kN}$

Tensile stress $=0.18 \mathrm{~N} / \mathrm{mm}^{2}$

Area of steel $=1130.97 \mathrm{~mm}^{2}$

\section{Ring beam at bottom:}

Size $=250 \times 500 \mathrm{~mm}$

Hoop tension $=262.95 \mathrm{kN}$

Area of steel $=1526.81 \mathrm{~mm}^{2}$

\section{Conical slab:}

Thickness of the slab $=200 \mathrm{~mm}$

Hoop tension $=579.36 \mathrm{kN}$

Area of steel $=2814.86 \mathrm{~mm}^{2}$

\section{Bottom dome:}

Thickness of the dome $=200 \mathrm{~mm}$

Meridional thrust $=92.09 \mathrm{kN}$

Hoop tension $=230 \mathrm{kN}$

Tensile stress $=0.374 \mathrm{~N} / \mathrm{mm}^{2}$

Area of steel $=600 \mathrm{~mm}^{2}$

\section{Circular girder:}

Size $=400 \times 600 \mathrm{~mm}$

Load $=4385.4 \mathrm{kN}$

Hoop stress $=1.5 \mathrm{~N} / \mathrm{mm}^{2}$

Hoop compression $=328 \mathrm{kN}$ 
Area of steel $=949.02 \mathrm{~mm}^{2}$

\section{Design and analysis in staad pro}

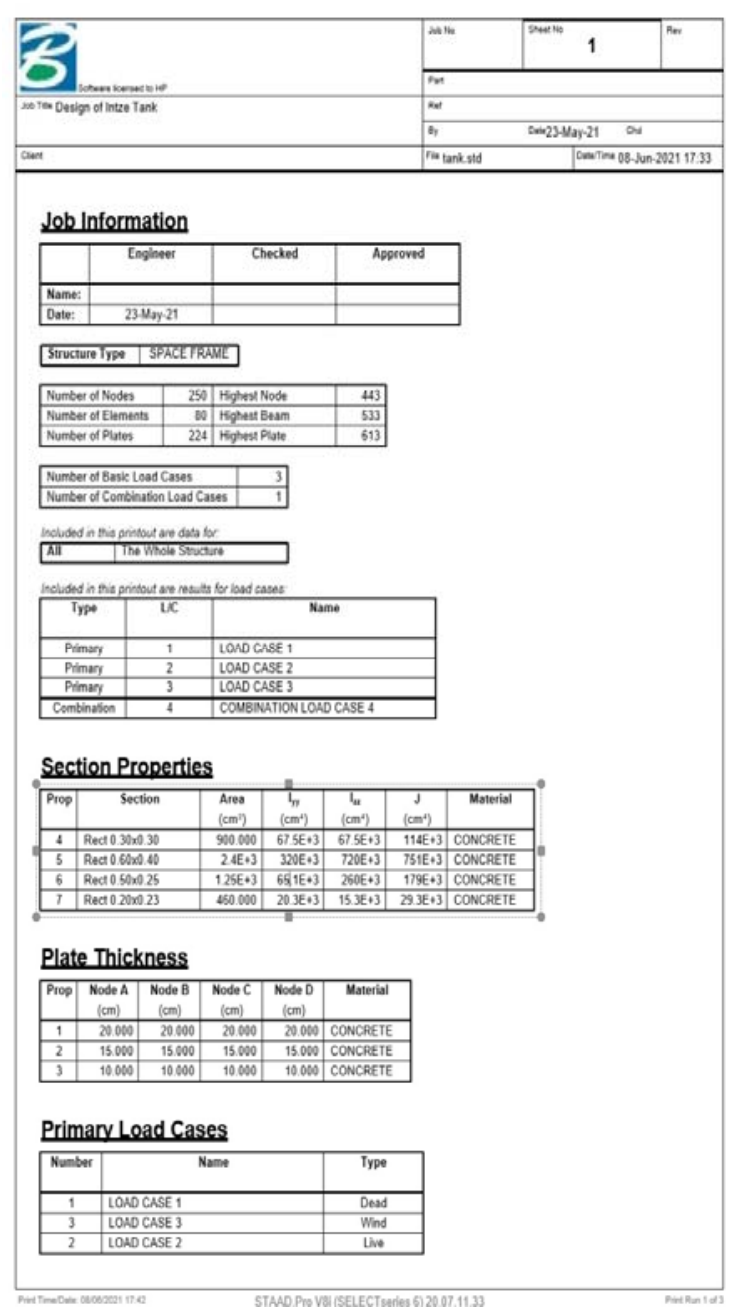

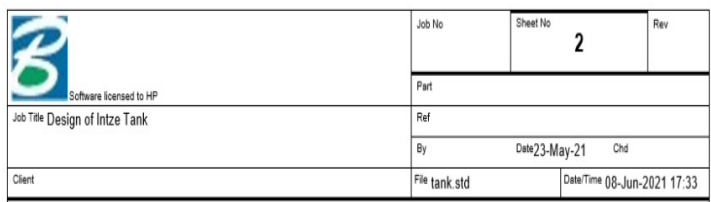

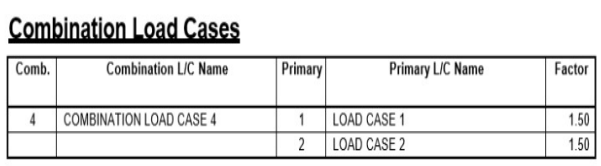

\section{Beam Displacement Detail Summary}

\begin{tabular}{|c|c|c|c|c|c|c|c|}
\hline & Beam & $\overline{U C C}$ & $\begin{array}{l}d \\
(m)\end{array}$ & $\begin{array}{c}x \\
(\mathrm{~mm})\end{array}$ & $\begin{array}{c}r \\
(m m)\end{array}$ & $\begin{array}{c}\mathrm{z} \\
(\mathrm{mm})\end{array}$ & $\begin{array}{c}\text { Resultant } \\
\text { (mm) }\end{array}$ \\
\hline$\overline{M a x X}$ & 483 & 4.COMBNATI & 0.000 & 0.488 & -7.621 & 0.000 & 7.637 \\
\hline Min. & 486 & & & 0.0489 & & & 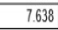 \\
\hline Max & 468 & $A D$ & 0.25 & 0.002 & U & 0.000 & 0.002 \\
\hline Min & 525 & 4.COMBII & 1.53 & 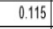 & 8.004 & 0.048 & 8.005 \\
\hline & & 4 & & & & & 7.638 \\
\hline Mn & 484 & 4:COMB & 3.0 & ${ }^{2}$ & -7.62 & 0.488 & 7.638 \\
\hline ax Rst & 533 & 4.COMBINATI & 1.531 & -0.116 & -8.004 & 0.48 & 8.0 \\
\hline
\end{tabular}

\section{Beam Force Detail Summary}

Sign corvention as diagrams- positive above line, negative below line except $F$ x where positive is compression. Distance dis given from

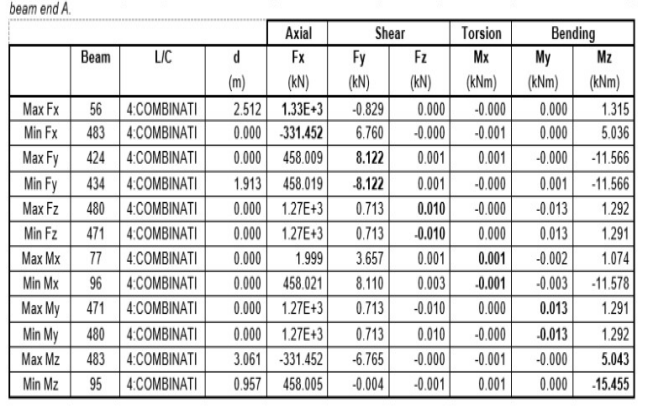

\section{Plate Center Principal Stress Summary}

\begin{tabular}{|c|c|c|c|c|c|c|c|c|}
\hline & & & Prin & & Von & & Tre & \\
\hline & Plate & LIC & $\begin{array}{c}\text { Top } \\
\left(\mathrm{N}^{2} \mathrm{~mm}^{2}\right)\end{array}$ & $\begin{array}{l}\text { Bottom } \\
\left(\mathrm{N} / \mathrm{mm}^{2}\right)\end{array}$ & $\begin{array}{c}\text { Iop } \\
\left(\mathrm{N} / \mathrm{mm}^{2}\right)^{2}\end{array}$ & $\begin{array}{l}\text { Bottom } \\
\left(\mathrm{N} / \mathrm{mm}^{2}\right)\end{array}$ & $\begin{array}{c}\text { Iop } \\
\left(\mathrm{N} / \mathrm{mm}^{2}\right)\end{array}$ & $\begin{array}{l}\text { Bottom } \\
\left(\mathrm{N} / \mathrm{mm}^{2} \mathrm{t}\right.\end{array}$ \\
\hline $\operatorname{Max}(t)$ & 438 & 4.COMBINATI & -2.728 & 0.684 & 5.111 & 1.495 & 5.896 & 1.715 \\
\hline $\operatorname{Max}(b)$ & 435 & 4COMEINATI & -2.728 & .0 .684 & 5.111 & 1.495 & 5896 & 1.715 \\
\hline $\operatorname{Max} V \mathbf{N}(t)$ & 435 & 4.COMBINATI & -2.728 & -0.684 & 5.111 & 1.495 & 5.896 & 1.715 \\
\hline $\operatorname{Max} V M(b)$ & 462 & 4.COMEINATI & 2.168 & 1.910 & 2.602 & 4.698 & 2.885 & 5.352 \\
\hline Tresca (t) & 435 & 4.COMEINATI & -2.728 & -0.684 & 5.1111 & 1.495 & 5.896 & 1.715 \\
\hline Tresca $(b)$ & 462 & 4.COMBINATI & 2.168 & 1.910 & 2.602 & 4.698 & 2885 & 5.352 \\
\hline
\end{tabular}




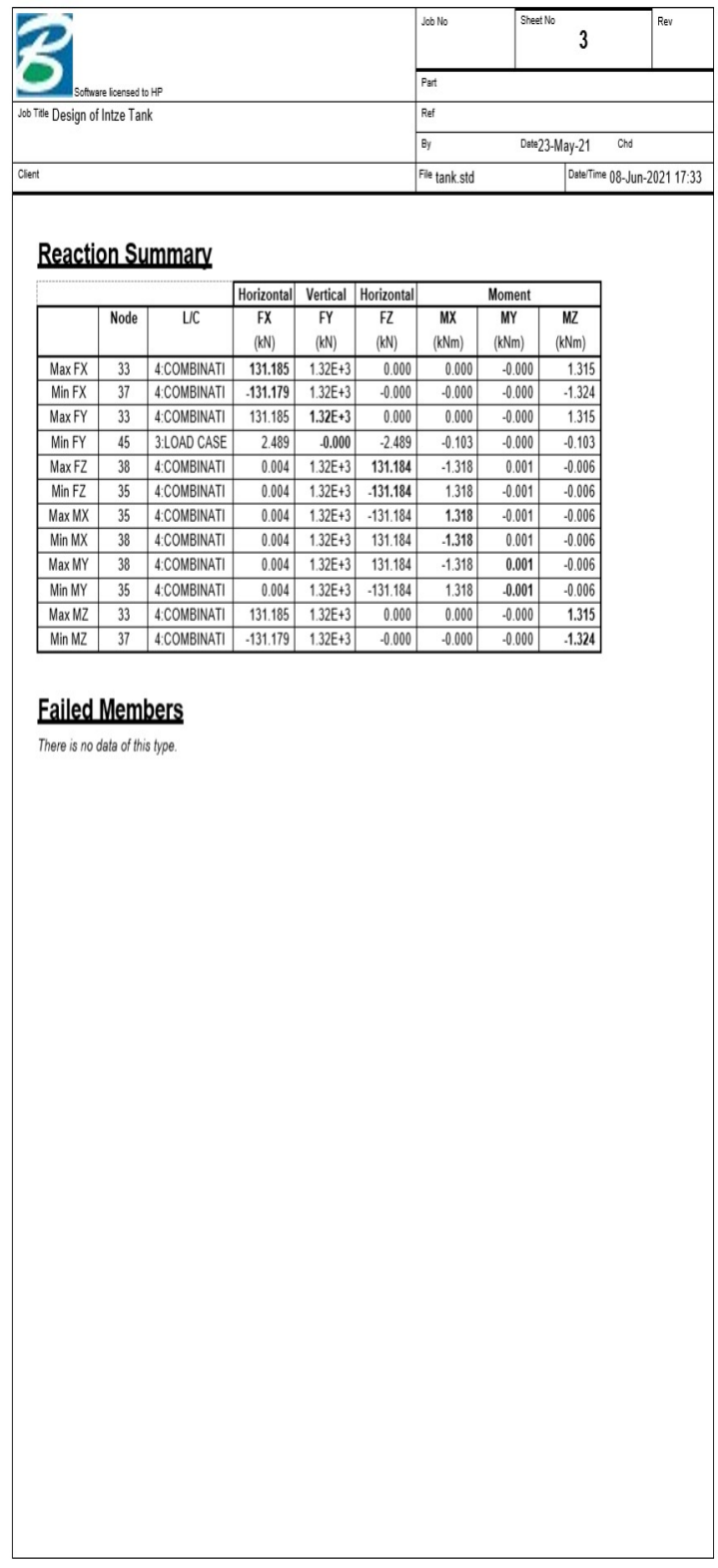

\section{Conclusion}

- An Intze water is designed with 300000 litres capacity with $12 \mathrm{~m}$ staging has designed with M30 grade of concrete.

- We design the tank by both manually and using STAAD Pro, the program results shown that design is safe

- After completion of Intze water tank design in STAAD Pro and from manual calculations we conclude that design is safe.

- Though design is safe but we observed that reinforcement is less when compared with manual calculations.

\section{References}

1. Text book: Design of Reinforced Concrete Structures by S. Ramamrutham.

2. I.S-3370 (Part IV-1967). Code of Practice for Concrete Structures for the storage of liquids.

3. I.S-3370 (Part II-1967). Code of practice for concrete structures for the storage of liquids.

4. Manchalwar, Atulkumar, and S. V. Bakre. International Journal of Dynamics and Control (2020): 1-10.

5. I.S:456-2000. Indian Standard Code of Practice for Reinforced Concrete.

6. I.S:875-1987 Part-III code design of wind loads.

7. Manchalwar, A., and S. V. Bakre. Soil Mechanics and Foundation Engineering 57.2 (2020): 170-177 